\section{US and Japan to cooperate in fusion and high energy physics}

A BROAD programme for collaborative research in a number of areas of energy science has been agreed in principle by the United States and Japan. The programme, which will include fusion research and high energy physics, is expected to involve expenditure of about $\$ 1,000$ million by the two countries over the next ten years.

The main areas of collaboration were agreed during a visit to Japan in September by Dr James Schlesinger, Energy Secretary, and Dr John Deutch, head of the Department of Energy's office of energy research. The details were worked out in a series of meetings held in Washington last week; and it is hoped that an agreement will be signed within four months.

The two main items in the programme are research into magnetic fusion, and into liquefaction of coal. In addition, the two sides have agreed to cooperate in solar photovoltaics, geothermal energy, and high energy physics.

As far as fusion is concerned, the main focus of Japanese interest will be the General Atomic Company's large Doublet III programme in San Diego, to which the Japanese are expected to contribute about $\$ 50$ million.

In addition, the two sides have agreed to set up a joint research institute for fusion research. The Japanese end of the institute will probably be in Nagoya; no decision has been reached on a US site, with Massachusetts Institute of Technology and the University of California at Los Angeles being possible contenders.

In return for being allowed to participate in the fusion programme, the Japanese have agreed to contribute $25 \%$ of the costs of a $\$ 700$ million coal conversion demonstration plant planned for construction by Gulf Oil in West Virginia. The German government has already agreed to contribute a further $25 \%$.

As for high energy physics, the two sides have in principle agreed on joint research and development into accelerator and particle detection technology and the joint construction and use of new facilities. It has been suggested for example that the Japanese may contribute to enhancing the $400 \times$ $400 \mathrm{GeV}$ intersecting storage accelerator (ISABELLE) at the Brookhaven Laboratory on Long Island. In the longer term, many US physicists are hoping that it may be possible to obtain joint funding for the next generation of particle accelerators.

Home insulation may increase \section{radiation hazard}

Scientists at the University of California's Lawrence Berkeley Laboratories have suggested that the reduced ventilation in private houses resulting from conservation measures may pose a potential health hazard, by increasing exposure to low levels of the radioactive gas radon

Radon-222 is produced as part of the decay chain of uranium-238. Both the gas and its short-lived decay "daughters"-in particular the alphaemitters polonium 218 and 214-are present in the natural environment and are a recognised source of background radiation. Furthermore radon's precursor, radium-226, is a trace element contained in most rocks and soil; and indoor radon sources therefore include many building materials.

The health hazard is caused principally by radon's daughters, which can attach themselves to airborne particles and if inhaled, can lodge in the lung and emit short-range alpha radiation. In recent years, the hazardous aspects of high-level radon exposure have been dramaticaly highlighted by the high incidence of lung cancer among uranium miners. And only recently the federal government has been taking steps to minimise public exposure to the radon emitted by uranium mill tailings.

Six scientists at the Lawrence Berkeley Laboratory's energy and environment division have now suggested that, if medical data from the uranium miners is extrapolated backwards-and assuming a nothreshold, linear dose-response relationship at low levels of exposure-the decreased ventilation in private homes achieved in the interests of energy conservation could theoretically lead to an extra 20 to 200 annual deaths per million population from lung cancer.

\section{New Spanish Constitution leaves science policy open}

ThE Spanish Parliament has just voted by a large majority (more than $90 \%$ ) for the second democratic Constitution of this century. On 6 December the Spanish people are called to vote the text in a referendum. The main political forces, from extreme left to neofrancoists, will campaign for its approval.

Science is mentioned twice in the first part of the new Constitution. Article 20 recognises "the right to literary, artistic, scientific and technical production and creation" and article 44 says that "the public powers will promote science and technical and scientific re-
The scientists point out that despite recent interest in the effects of radon exposure in both Sweden and the UK, there is still little detailed knowledge about the effects of low level exposures over long periods of time, and that any conclusions are highly speculative. "We are not issuing a warning, but merely calling attention to a possibility that should be watched and studied", says Dr Craig Hollowell, one of the scientists concerned.

Despite this, a report produced by the scientists states that: "It is likely that some increased lung cancer risk would result from increased radon exposures; hence it is desirable not to allow radon concentrations to rise significantly".

In the long term, the scientists suggest that it may be necessary to include radon exposure levels in building standards. In the short term, they suggest a number of measures - such as sealing walls and floors, or coupling mechanical ventilation with heat exchangers-to reduce radon exposure.

The scientists have applied to the Department of Energy for further funds to carry out a more detailed investigation of the potential hazardsa request which has fallen between the department's environmental health and conservation responsibilities.

Some money has already been granted to study the radon emission of building materials. A complementary proposal for studying the health effects is being considered by the department's office of energy research. No one is yet prepared to admit that increased radon exposure does pose a health hazard, however small; but with growing concern about the long-term effects of low level radiation, no one is yet prepared to dismiss the hypothesis as unfounded.

David Dickson search for the benefit of general interest".

The mention of research in the articles dealing with the organisation of the State may be of greatest significance for scientists. One of the central constitutional problems has been the formation of autonomous governments in different parts of Spain. Article 148 lists the matters transferable to autonomous communities and it includes "the promotion of culture and research". However "the promotion and general coordination of technical and scientific research" can be found in the list of matters reserved 